\title{
Is the Network Capable of Computation?
}

\author{
Peter Perešíni \\ EPFL \\ peter.peresini@epfl.ch
}

\author{
Dejan Kostić \\ Institute IMDEA Networks \\ dkostic@imdea.org
}

\begin{abstract}
Ensuring correct network behavior is hard. Previous state of the art has demonstrated that analyzing a network containing middleboxes is hard. In this paper, we show that even using only statically configured switches, and asking the simplest possible question - "Will this concrete packet reach the destination?" - can make the problem intractable. Moreover, we demonstrate that this is a fundamental property because a network can perform arbitrary computations. Namely, we show how to emulate the Rule $\mathbf{1 1 0}$ cellular automaton using only basic network switches with simple features such as packet matching, header rewriting and round-robin loadbalancing. This ultimately means that analyzing dynamic network behavior can be as hard as analyzing an arbitrary program.
\end{abstract}

\section{INTRODUCTION}

Correct network behavior is often taken for granted, but ensuring it is a daunting task. It was previously shown that statically analyzing the network and answering questions such as "can host A talk to host B" can be NP-hard [11].

The networks are increasing in complexity, as well as in the set of roles they are supposed to fulfill. Today's computer networks consist of equipment such as switches/routers ${ }^{1}$, and various kinds of middleboxes (load-balancers, intrusion detection systems, network address translators, etc.). Each of these devices exposes traffic to a variety of features (packet matching, packet rewriting, etc.). These features may, however, interact in very complex ways. This means that analyzing dynamic network behavior (which cannot be checked statically) is also becoming more and more difficult.

For example, in security, protocol interactions can pose significant security risks amongst otherwise secure protocols [9]. Currently, there is a lack of similar research on the interaction of basic features of today's networks. This gap is becoming more important as software-defined networking, mainly the OpenFlow protocol, is growing up in its popularity.

In this paper, we show that the complexity of the interactions is inherent even in simple scenarios. We investigate the interaction between four simple network mechanisms - a packet header matching, a packet header rewriting, unicast/multicast forwarding and round robin load-balancing. On its own, each of these mechanisms is fairly simple but their combination might easily become hard to analyze.

\footnotetext{
${ }^{1}$ In this paper, we will use the terms switch and router interchangeably, both meaning a device capable of matching packets according to some criteria and forwarding them to one or more destinations.

978-1-4799-1270-4/13/\$31.00 (C) 2013 IEEE
}

\section{A. Let me count it for you!}

To illustrate that simple switch features might interact in a complex way, we construct a binary counter i.e. a device which can go through states $0,1,10,11,100,101, \ldots$ We use a single switch with an ingress, egress and loopback ${ }^{2}$ link supporting following features: $(i)$ matching packets on (a combination of) header fields (i.e., either exact match or wildcard for each field); (ii) support of different match priorities (for overlapping match rules); and (iii) rewriting packet headers and/or forwarding to a specific port based on the matched rule.

We represent the value of the counter as a binary number where each bit is stored in its own packet header field. ${ }^{3}$ When a packet enters on the ingress port, we clear the packet by rewriting all applicable header fields to zeroes. The increment by one operation is performed by matching the longest suffix " $0,1,1,1, \ldots, 1,1,1$ " of header fields, rewriting it into " $1,0,0,0, \ldots, 0,0,0$ ", and looping the packet. There are two special cases: $(i)$ all header fields are 0 - we rewrite the last field to 1 and loop; $(i i)$ all header fields are 1 - we forward the packet to the output and finish the counting. The rules are summarized in Figure 1.

Our example shows that we can loop the packet for an exponential time just by using a single switch with a single loopback link - If the packet header contains $n$ independent fields, we can loop the packet $2^{n}$ times using just $n+2$ rules. Moreover, we can simply extend the example and emulate $k$-ary counters, counting up to $k^{n}$ by using only $\Theta(n * k)$ rules. Such complicated packet behavior may definitely pose a formidable opponent to the static network analyzers such as header-space analysis [8]. We therefore ask the question "Is it even possible to analyze a network consisting of few elements, each one with simple but dynamic forwarding behavior?".

\section{B. Contributions}

The core contribution of this paper is a description how to build reusable boolean gates and memory buffers using just a set of network devices with simple features such as packet matching, header rewriting and round-robin load-balancing. Furthermore, we demonstrate the use of these gates to efficiently emulate Rule 110 cellular automaton [14] which is the first step towards efficiently emulating tape-bounded Turing machines. By providing an efficient emulation of Rule 110, we

\footnotetext{
${ }^{2}$ e.g. 2 switch ports connected to the same link

${ }^{3}$ It might not be possible to use all available header fields - for example by clearing protocol field one invalidates all IPv4 fields
} 


\begin{tabular}{|c|c|c|c|c|}
\hline \multirow{2}{*}{ rule } & \multicolumn{2}{|c|}{ match } & rewrite & fwd \\
\cline { 2 - 5 } & port & $H_{n} \ldots H_{1}$ & $H_{n} \ldots H_{1}$ & \\
\hline \hline init & in & $\star \star \star \star \star \star \star$ & 0000000 & loop \\
\hline finish & loop & 1111111 & ----- & out \\
\hline \hline digit $n$ & loop & 0111111 & 1000000 & loop \\
\hline digit $n-1$ & loop & $\star 011111$ & -100000 & loop \\
\hline digit $n-2$ & loop & $\star \star 01111$ & -10000 & loop \\
\hline digit $n-3$ & loop & $\star \star \star 0111$ & --1000 & loop \\
\hline digits $n-4$ to 3 & \multicolumn{3}{|c|}{$\ldots$} & \multicolumn{2}{|c}{$\ldots$} \\
\hline digit 2 & loop & $\star \star \star \star \star 01$ & --10 & loop \\
\hline digit 1 & loop & $\star \star \star \star \star \star 0$ & ----1 & loop \\
\hline
\end{tabular}

Fig. 1: Binary $n$-digit counter. Rules are in the order of deacreasing priority. "*" denotes a wildcard match and "-" denotes no rewrite of the corresponding header field.

conjecture that providing the answer to the simplest possible question - "Will this concrete packet reach the destination?" - can be as hard as analyzing arbitrary computer programs.

Therefore, the main contribution of this paper does not entail a new approach for ensuring network correctness. Instead, it is a fundamental result suggesting the nonexistence of general dynamic network analyzers.

\section{MODELING THE NETWORK \& BASIC BUILDING BLOCKS}

In this section we informally introduce a network model used through this paper. For the formal model, please refer to the appendix in our Technical Report [13]. Additionally, we present basic building blocks we use in the rest of the paper, each building block corresponding to a statically configured switch.

Our goal is to model an asynchronous computer network with unbounded propagation time. The model of the network is represented as a directed graph with nodes representing switches and edges representing directed links. For the modeling purpose, we abstract out the packet delivery over links. Instead, we assume that each packet in the network is located either at an ingress or at an egress queue of some switch. We assume only simple FIFO queuing disciplines and atomic packet processing, that is, the network state can change by (i) atomically moving the first packet from a switch egress queue to the connected switch's ingress queue (i.e. sending the packet over a link); (ii) atomically moving the first packet from a switch ingress queue, enqueuing it (with possible modifications) to all relevant egress queues and updating switch state (i.e. switch packet processing).

From the switches, we require support for the following packet processing capabilities:

Forward/multicast: The switch can be configured such that any packet on one of its ports will be forwarded to one or several other ports.

Header matching: The switch is able to match a packet on a combination of header fields and forward it to the port associated with the matching rule. We require support for overlapping rules with different priorities.

Header rewriting: The switch is able to rewrite a subset of header fields and then forward the packet.

Round-robin load-balancing: The switch provides a loadbalancing mechanism where the packet on the ingress port is forwarded to one of the $m$ egress ports. The output port is chosen in a round-robin fashion, where the first packet is forwarded to the first egress port, the second packet forwarded to the second egress port, ... the $m+1$-th packet forwarded again on the first egress port.

Although real switches might support any combination of these features, it is enough for us to have just a single capability per switch. We use the switches with such capabilities to model basic building blocks for our Turing machine construction. We will call these building blocks gates (summarized in Fig. 2) and each basic gate is easily implemented by a single switch. We leave possible optimization of merging several of these elements into a single switch as a future work. The gates we use are as follows:

split: Copies a packet from one ingress link (also denoted as input wire) to multiple output wires by using the multicast capability.

merge: Accepts a packet on any input wire and forwards it to the output.

rrobin: Packets on any input port should be forwarded to one of several output wires in a round-robin fashion. The element is implemented by a round-robin load-balancer feature.

rewrite: Rewrite packet header field(s) of any incoming packet with the new values and forward it to the output.

conditional: Emulate a simple decision-making process using the packets by outputting the packet to one of the outputs depending on the match. The element is realized as a switch with two rules: $(i)$ a high-priority yes rule matching the condition on the header field; (ii) a low-priority no rule that matches everything else (default rule).

join: The join ${ }^{4}$ is an element which filters a sequence of packets and passes through only each $m$-th packet on the input. It is implemented as rrobin with first $m-1$ egress ports "dead" (not connected to any link).

\section{BOOLEAN FUNCTIONS}

As a first step towards a Turing machine emulator, we show a way to emulate a boolean circuit [1] - an acyclic directed graph with $n$ nodes with not incoming edges called inputs, $m$ nodes with no outgoing edges called outputs and several other nodes called logical gates. We will call all edges of a boolean circuit wires because of their representation in hardware circuits. Each logical gate contains several incoming edges, a single outgoing edge and performs a boolean function over its inputs. In this paper, we will consider only logical gates AND, OR and NOT as they are functionally complete and one can construct any other logical gate out of them - in fact, AND and NOT would be sufficient [7].

\footnotetext{
${ }^{4}$ similar to join in UML diagrams
} 


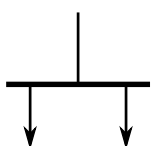

(a) split (copy to multiple destinations)

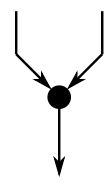

(b) merge multiple sources

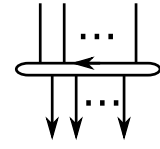

(c) round robin (loops through $m$ outputs)

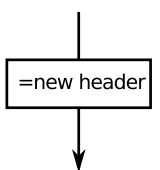

(d) header rewrite (sets new packet header)

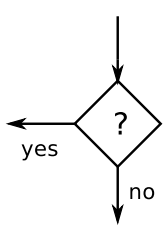

(e) conditional

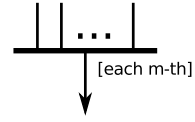

(f) join (pass through every $m$-th packet)

Fig. 2: Pictograms of basic building blocks, each one can be implemented by a single switch.

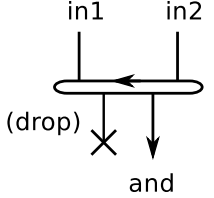

(a) AND

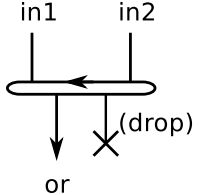

(b) OR
Fig. 3: Basic boolean operators (single-wire inputs)

\section{A. Representing boolean circuits as computer networks}

We represent each logical gate as a set of interconnected switches (e.g., basic elements). Edges of the boolean circuit graph are represented by network links. Although boolean circuits use bits as input values, we cannot represent bits by a packet header field - both AND and OR need in certain situations to wait for both inputs before they can produce the output. However, such "packet save" functionality is not present in the switches. Instead, we take an approach similar to the hardware implementation of boolean circuits and we represent boolean values as the presence/absence of a single packet on the link.

\section{B. Simple and and or gates}

For both and and or, we exploit the fact that rrobin element cycles through its outputs. The key idea is simple and needs to produce a single packet on the output iff (if and only if) it receives packets on both of its inputs. That is, we need to drop the first packet (if any) and let through the second packet (if any). This is a perfect match for a rrobin gate with its first output link dead ${ }^{5}$ and the second output representing the and output as illustrated in Fig. 3. Note that while the and gate has the same hardware implementation as the join $(m=2)$ gate, we will use two gates to make the semantic distinction. The or can be realized in a similar manner as the and, the only difference is that we exchange the live and dead links.

\section{Negations}

This approach, as is, cannot handle negations. The not gate would need to produce a packet iff there is no (and will not be in the future!) packet on the input. This is simply impossible to achieve in asynchronous networks with unbounded propagation time. To address this issue, we develop a two-wire bit representation. In this representation, each input

\footnotetext{
${ }^{5} \mathrm{We}$ assume that round-robin load-balancing algorithm in the switch does not skip over dead links. Otherwise, we may utilize an additional switch instructed to drop any incoming packet
}

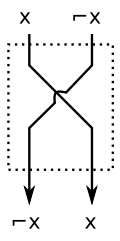

(a) NOT

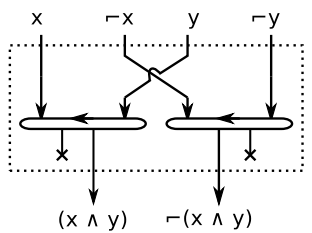

(b) AND

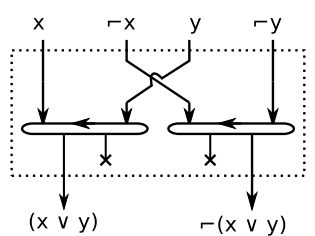

(c) OR
Fig. 4: Boolean operators using the two-wire representation.

of a boolean circuit consists of two network links, each link corresponding to one of the two possible bit values. The boolean circuit input is represented as a single packet on exactly one of the wires. If the value of input $x$ is one, the input consists of a single packet on the wire denoted " $x$ ". Otherwise (the value is zero), the input consists of a single packet on the wire denoted " $\neg x$ ".

The not gate in this new representation is simply an exchange of the wires (Fig. 4a). For the and and or gates, we reuse the previously introduced single-wire and/or gates and combine them using De Morgan identities $\neg(x \wedge y)=$ $(\neg x) \vee(\neg y), \neg(x \vee y)=(\neg x) \wedge(\neg y)$ to produce the two-wire gates (Fig. 4b, 4c) [6].

\section{REPEATING COMPUTATIONS - REUSING GATES MULTIPLE TIMES}

If we want to emulate a (possibly exponentially long) computation of a Turing machine with a network of polynomial size, we need to reuse the same gates several times with different inputs. This, however, poses a new challenge - we need to clear/reset the internal state of these gates. In particular, the elements needed to be reset are all rrobin elements as they end up in different states depending on the number of input packets. Additionally, we need a mechanism for slowing down some packets - sending a second set of inputs to the gates too fast can end up with some of the new packets interfering with the previous, still-running, computation. In this section, we address these two challenges by designing clearable gates and a new special element which can "buffer" packets.

\section{A. The art of clearing}

In order to reuse gates, we need to reset any changes to the internal state of our gates. This is trivial for a not gate, but poses a significant challenge to and and or gates, which contain state of the round-robin algorithm inside. To address this, we introduce a special clearing packet(s) that can be distinguished from information packets by matching on some header field.

To illustrate the mechanism of clearing, consider the and 


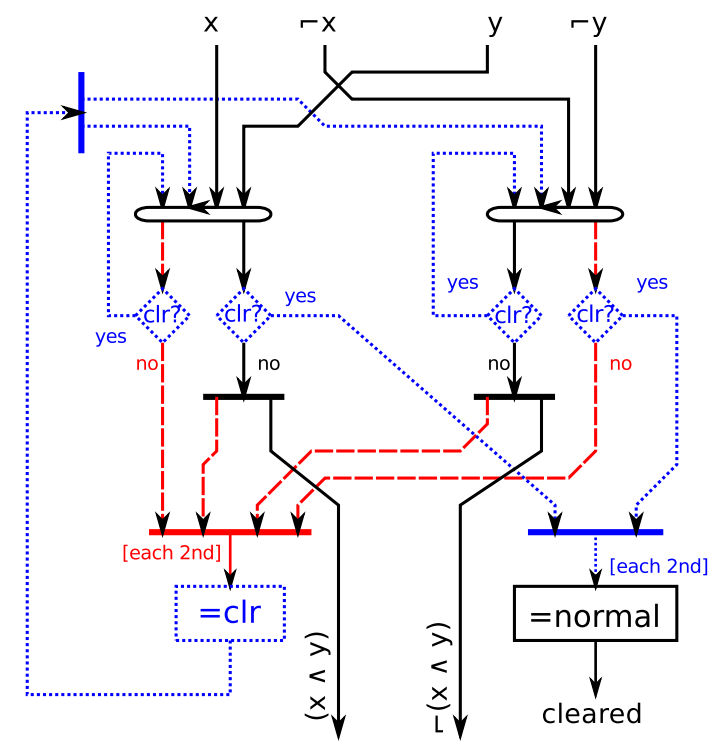

Fig. 5: Clearable AND gate. Original AND is in black, red part (dashed) waits until the circuit finishes computing and blue (dotted) part is responsible for clearing.

gate from Fig. $4 \mathrm{~b}-$ we extend this gate to a self-clearing and gate in Fig. 5. In the new gate, the clearing packet needs to be created after the original rrobin gates finished the computation, i.e. processed all packets on their inputs. As we know that there will be exactly two input packets in total on four input wires, we can identify this situation by $(i)$ (red part of Fig. 5) collecting notifications - (copies) of all packets output by rrobin elements representing AND/OR, (ii) (blue part of Fig. 5) start clearing after we receive all notifications. As there will be exactly two notifications on the four notification links, we introduce new join element which passes each second packet. Note that the newly introduced join internally contains a new rrobin which potentially must be reset. This rrobin is, however, reset to its initial state after receiving the second packet and we do not need to clear it explicitly.

The output of the join is then converted into a clearing packet by header rewriting. We split this clearing packet to clear both rrobin elements in parallel. A single rrobin element can be easily reset to its initial state by processing a specific number of packets. We therefore employ a looping trick where we loop the clearing packet enough times that after the last iteration, the element will be in the initial state. We do this by intercepting rrobin outputs and looping the clearing packet on all but the last rrobin output link.

Finally, after both clearing packets for both rrobins finish looping, we wait for the last one by using join. The result is a single packet indicating that the circuit was cleared. Again note that newly introduced join does not need an explicit reset.

Observation: Clearing does not interfere with the computation. Clearing starts after both rrobin elements finish processing their inputs. The rest of the computation (i.e. forwarding packets to the output) is unaffected by parallel cleaning as there are no more elements with the internal state.

Observation: Clearing eventually finishes and when the circuit outputs a signal, it is ready to be reused. After receiving all (two) input packets, they will be eventually forwarded to join and thus eventually reach the clearing rewrite. Subsequently, the newly split packets will both loop a finite number of times before being forwarded to the final join. By looping, these packets will reset both rrobin elements and join elements self-reset right after they place a packet to their output queue (and before the packet is forwarded further).

\section{B. Waiting - because clearing is not enough!}

To correctly repeat calculations, clearing the state is not enough because the circuit is quite brittle to race-conditions if we send new inputs while the circuit is clearing, the clearing can interfere with the ongoing computation. Therefore, we need an element which can hold the inputs till we know it is safe to send them further. To address this, we create a new buffer element. The challenge is to perform the buffering task without any direct control over switch buffers - the only way to prevent a packet from being processed by the switch and sent on the output link is to drop it.

The key idea behind the buffer element (Fig. 6) is that we actually drop packets and then "recreate" them when needed. Of course, we are not able to fully recover the original packet with all headers and data. However, this is not needed - we can simply copy any other existing packet as in our case only the presence or absence of a packet matters ${ }^{6}$. The building block of the buffer element are two join elements, one for each input wire $(x, \neg x)$. Both of these joins are wait for two incoming packets - the input packet and a packet on the signal wire telling that the buffer can be released. Upon receiving both the input and the signal packet (in any order), exactly on of the joins will let through last of these packets. At this state we $(i)$ release the buffer by sending this packet to the corresponding output; $(i i)$ reset clear the buffer. Here, we again utilize the special clearing packet. However, depending on which half of the circuit needs to be cleared, we separate the two scenarios by using a different header for the clearing packet as displayed in blue/red color in Figure 6.

Observation: Buffer does not pass any packet until it receives both the input and the signal. Trivially, a single packet (either the input or the signal) is not able to pass any of the join elements.

Observation: Buffer is cleared properly and signals its clearing only when it is safe to reuse it. We will use the fact that packets following the same path cannot be reordered (they cannot be reordered on links and we assume switches with FIFO input/output port queues).

Without a loss of generality, assume an input packet on wire $x$. After receiving packets on both $x$ and signal, the join (2) will pass through the later packet while join (3) will drop the signal. Subsequently, the passed packet continues to

\footnotetext{
${ }^{6}$ Actually, it is also important that the packet is created as information and not clearing packet. We copy only information packets.
} 


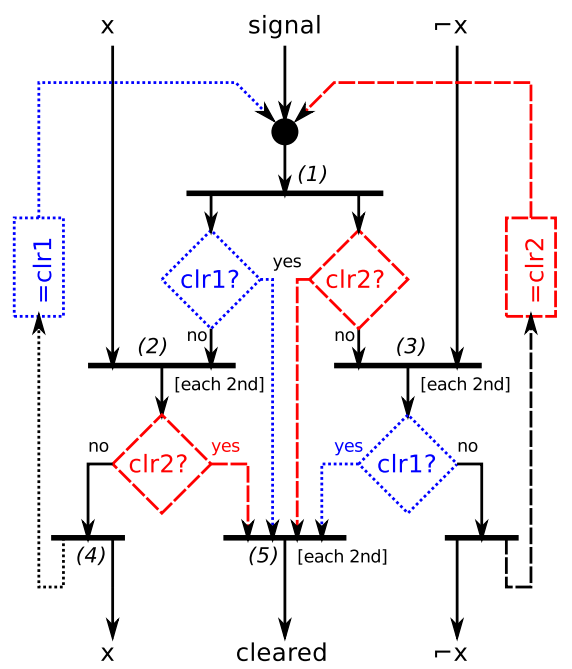

Fig. 6: Buffer (holds value until a signal is received). Depending on the input, either blue (input $=x$ ) or red (input $=\neg x$ ) clearing part is used.

split (4) where it is forwarded to the output and looped back as a clearing packet. The clearing packet is then split again at (1) with one part (left branch) being sent to join (5) and the second part (right branch) going resetting join (3) and further looping before finally reaching join (5).

Notice that for buffer functioning properly, the clearing packet (right branch) must reach input join (3) after the signal packet. If we simply looped directly from (4) to (3) and bypassed (1), the signal could take too long to travel from (1) to (3) and the clearing packet would be there first. Instead, our trick is to use merge and then split to guarantee that at the time we created the clearing packet, the original signal packet must have reached split (1) and the clearing packet will be placed behind it on all FIFO queues towards join (3).

\section{FROM CIRCUITS TO CELLULAR AUTOMATA}

The final step in showing the complexity of possible network computation is to show that clearable logical gates and buffer are sufficient to emulate Rule 110 cellular automaton.

Rule 110 Rule 110 [14] is a simple linear cellular automaton (an array of cells) with each cell holding a binary value. Rule 110 computation consists of discrete steps, each step synchronously updating values of all cells. In each step, each cell looks at its own value and the values of its neighbors and updates its value according to the function from Fig. 7. Although Rule 110 is a simple automaton, it has been shown that it is capable of emulating the first $n$ steps of a Turing machine in a polynomial number of cells [12]. Thus, by emulating Rule 110 using a polynomial number of network elements, we will be able to emulate the first $n$ steps of a Turing machine in polynomial network size. This will prove that not only networks are capable of emulating Turing machines but that the emulation is also semi-efficient.

Rule 110 emulator We observe that the Rule 110 function in Fig. 7 can be translated into a boolean circuit as $f\left(x_{i-1}, x_{i}, x_{i+1}\right)=\left(\neg x_{i-1} \wedge x_{i+1}\right) \vee\left(\neg x_{i} \wedge x_{i+1}\right) \vee\left(x_{i} \wedge\right.$ $\left.\neg x_{i+1}\right)$. Thus, by composing clearable logical gates, we can

\begin{tabular}{c|c|c||c}
$x_{i-1}$ & $x_{i}$ & $x_{i+1}$ & new $x_{i}$ \\
\hline 0 & 0 & 0 & 0 \\
0 & 0 & 1 & 1 \\
0 & 1 & 0 & 1 \\
0 & 1 & 1 & 1 \\
1 & 0 & 0 & 0 \\
1 & 0 & 1 & 1 \\
1 & 1 & 0 & 1 \\
1 & 1 & 1 & 0
\end{tabular}

Fig. 7: Rule 110 - a simple linear automaton

create a clearable rule110 gate.

We create Rule 110 automaton as an array of interconnected circuits, each circuit representing one cell of the automaton. Each circuit is composed of a buffer holding the input values, the rule110 gate and the output value buffer. ${ }^{7}$ The inputs of rule110 gate are connected to input buffers of the current and the neighboring cells, while the output is connected to the output buffer. Finally, to enable the multi-step computation, we send the values of output buffers back to the input buffers and thus create a loop. The overall structure of the construction is shown in Fig. 8.

Observation: The emulator construction in Fig. 8 emulates the Rule 110 cellular automaton in synchronous steps.

It is easy to see that the circuit is iteratively computing Rule 110 cell values from the previous values. What is more challenging is the proof that cells are updated synchronously and that we do not feed values to the circuit before it acknowledged clearing. We use the natural split of the circuit into three stages: $(i)$ input buffers; ( $i i)$ Rule 110; and (iii) output buffers. When the computation begins, all packets are in stage $(i)$. After receiving the "start" packet, the computation can proceed to stages $($ ii) and $(i i i)$. However, the output buffers in stage (iii) will hold all packets until all input buffers and all rule110 circuits acknowledge clearing. At this moment there are no packets in stage $(i)$ and $(i i)$ - the input buffers and rule110s are cleared. Thus, the output buffers can forward the packets back to the input (stage $(i)$ ). Here, the packets will be held until all output buffers acknowledge clearing and there is not packet in stage $(i i i)$. Only after this, the new round of computation may begin. Thus, we proved that the circuit is updated synchronously and that there is no unwanted interference between the packets in different stages.

\section{FUTURE WORK}

There is certainly room for improving our results. In particular, we believe that using the similar idea as in Rule 110 emulator, it is possible to construct an emulator of tape-bounded Turing machines. Such emulator, if constructed, would be capable of computing exponential number of steps in polynomial network size, achieving efficient emulation. We will leave this construction as a future work.

\footnotetext{
${ }^{7}$ We need to have two sets of buffers (input and output) because the computation is only loosely synchronized and we do not want results to interfere with inputs (e.g., by receiving new buffer value before the buffer is completely cleared).
} 


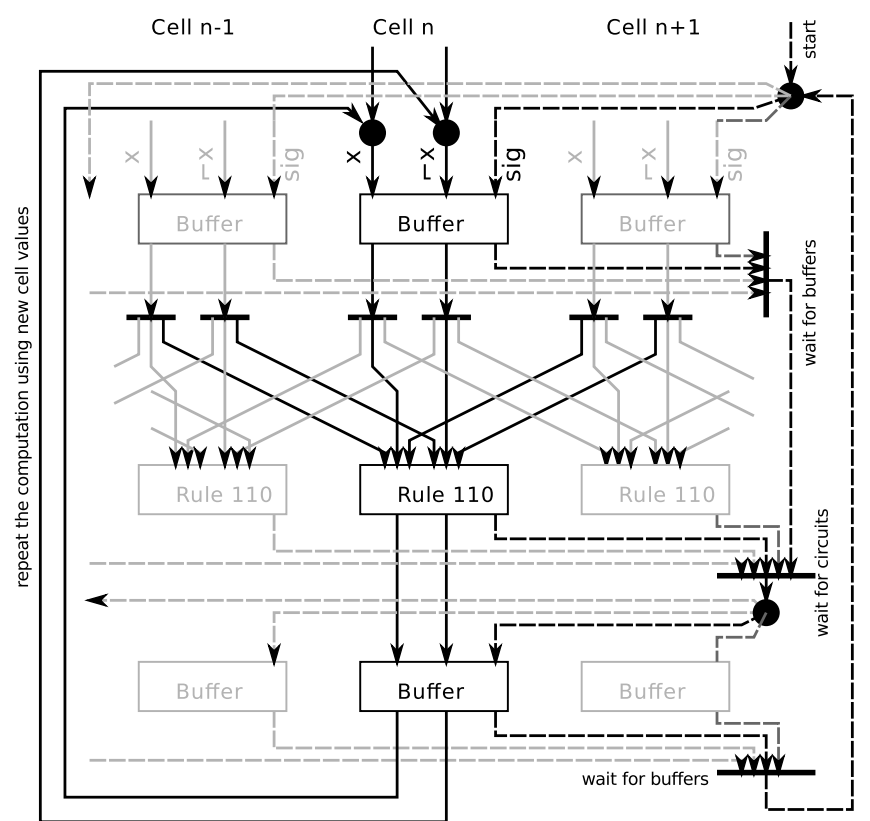

Fig. 8: Rule 110 emulator (showing only cells $n-1, n$, and $n+1)$. Black part is responsible for one step of computation of cell $n$, grey parts represents different cells. Dashed part is responsible for synchronizing and advancing the computation.

Another area of potential future work is optimization of the created network - in the current construction one switch for each single element. This unnecessarily wastes resources because switches typically have many ports and complicated packet processing pipelines. It is therefore interesting to explore this area to provide much smaller building blocks (e.g., by packing one whole Rule 110 cell into a single switch with several loopback links).

Finally, the constructs we show in this paper are quite brittle - they heavily depend on no other packets being forwarded by the switch. It is an interesting future direction to see if we can add some robustness to the design.

\section{RELATED WORK}

We are certainly not the first to suggest that systems with simple features might interact in a complex way. Protocol interaction from a security viewpoint was analyzed in [9]. Feature interaction was also studied in telecommunication services [3], [4]. Unlike these studies, we are not trying to come up with a solution to the feature interaction problem. Indeed, we take an opposite way - we are trying to exploit the interaction of simple features and show that in general it might be very hard to analyze.

Parasitic computing [2] demonstrates the potential of solving SAT by network checksumming. Implicit simulations using messaging protocols [10] demonstrate the use of the ICMP protocol as another way to perform simple computations using the network. Both [2] and [10], however, need a host which creates the packet and the network works just as a simple filter of results. In contrast, we do not require intelligent endhosts, and still perform Rule 110 emulation in the network. Contem- poraneously, Chiesa et al. show that BGP configurations can emulate logic circuits [5].

The problem of analyzing the network behavior was tackled by [15], [11]. These tools, however, perform only a static analysis of the network. They are not able to analyze the dynamic behavior of round-robin loadbalancers. Instead, for the analysis, they overapproximate the loadbalancing primitive simply as a multicast. Our result implies that this overapproximation trick enables them to predict the network behavior much faster than a dynamic analyzer could do, albeit at the cost of precision.

\section{CONCLUSIONS}

It is common wisdom that debugging networks is hard analyzing a network containing middleboxes is commonly understood to be hard. In fact, it was previously shown that statically analyzing a network composed just of switches and answering questions such as "can host A talk to host B" can be NP-hard [11]. In this paper, we further move the boundary and show that the ability to analyze the dynamic behavior of statically configured networks consisting only of simple switches is equivalent to the ability of analyzing outcome of a cellular automaton computation. We therefore conjecture that a dynamic analysis of networks can be an intractable problem.

\section{ACKNOWLEDGMENTS}

The research leading to these results has received funding from the European Research Council under the European Union's Seventh Framework Programme (FP7/2007-2013) / ERC grant agreement 259110.

\section{REFERENCES}

[1] S. Arora and B. Barak. Computational Complexity: A Modern Approach. 2007. http://www.cs.princeton.edu/theory/complexity/circuitschap.pdf.

[2] A.-L. Barabasi, V. W. Freeh, H. Jeong, and J. B. Brockman. Parasitic computing. Nature, 412(6850):894-897, Aug. 2001.

[3] T. Bowen, F. Dworack, C. Chow, N. Griffeth, G. Herman, and Y.-J. Lin. The feature interaction problem in telecommunications systems. In SETSS, 1989

[4] M. Calder, M. Kolberg, E. H. Magill, and S. Reiff-Marganiec. Feature interaction: a critical review and considered forecast. Computer Networks, 41(1):115 - 141, 2003.

[5] M. Chiesa, L. Cittadini, G. D. Battista, L. Vanbevery, and S. Vissicchioy. Computing with bgp: from routing coniňAgurations to turing machines. Technical report, 2012

[6] I. M. Copi and C. Cohen. Introduction to logic. MacMillan, 1990.

[7] H. Enderton. A Mathematical Introduction to Logic, Second Edition. Harcourt/Academic Press, 2001.

[8] P. Kazemian, G. Varghese, and N. McKeown. Header space analysis: Static checking for networks. In NSDI, 2012.

[9] J. Kelsey, B. Schneier, and D. Wagner. Protocol interactions and the chosen protocol attack. In Proceedings of the 5th International Workshop on Security Protocols, 1998.

[10] G. Kohring. Implicit simulations using messaging protocols. International Journal of Modern Physics C, 14(2):203-213, 2003.

[11] H. Mai, A. Khurshid, R. Agarwal, M. Caesar, P. B. Godfrey, and S. T. King. Debugging the data plane with anteater. In SIGCOMM, 2011.

[12] T. Neary and D. Woods. P-completeness of cellular automaton rule 110. In ICALP, 2006.

[13] P. Peresini and D. Kostic. Is the network turing-complete? Technical report, EPFL, 187131, 2013.

[14] S. Wolfram. Theory and Applications of Cellular Automata. World Scientific, 1986.

[15] G. Xie, J. Zhan, D. Maltz, H. Zhang, A. Greenberg, G. Hjalmtysson, and J. Rexford. On static reachability analysis of ip networks. In INFOCOM, 2005. 